\title{
新型吡唑基取代的甲磺酰基类化合物的合成及除草活性研究
}

\author{
温彦鹏 $a$ 张 爽 $b$ 侯广峰 $b$ 于颖慧 $*, a, b$ 高金胜*,a,b \\ ( ${ }^{a}$ 黑龙江大学化学化工与材料学院 哈尔滨 150080) \\ $\left({ }^{b}\right.$ 黑龙江大学农药工程中心 哈尔滨 150080)
}

\begin{abstract}
摘要 为获得具有新颖结构和较高药效的新型除草剂, 基于环磺酮的结构, 对环磺酮结构中苯环的间位取代基及环己 二酮环进行了修饰替换，设计了 6 个新型的含有吡唑基的甲磺酰基类杂环化合物. 以 2-甲基-3-氯苯胺起始原料，经重 氮化、乙酰基化、两步氧化、酯化、溴化、亲核取代、氯化、缩合等 9 步反应最终获得 6 个目标化合物. 最终产物以 及重要中间体的结构采用 ${ }^{1} \mathrm{H} N \mathrm{NM} 、{ }^{13} \mathrm{C} \mathrm{NMR} 、$ FTIR、元素分析等方法进行表征与确认. 对该 6 个化合物的除草活性 进行了测试, 结果表明各化合物对芥菜、繁缕、看麦娘、小藜、棒头草均表现出良好的除草效果.
\end{abstract}

关键词 吡唑基; 甲磺酰基; 环磺酮; 合成; 反应条件; 除草活性

\section{Syntheses and Herbicidal Activities of New Methylsulfonyl Compounds with Pyrazol Group}

\author{
Wen, Yanpeng ${ }^{a} \quad$ Zhang, Shuang $^{b} \quad$ Hou, Guangfeng ${ }^{b}$ \\ Yu, Yinghui*,a,b Gao Jinsheng ${ }^{*, a, b}$ \\ ( ${ }^{a}$ School of Chemistry and Materials Science, Heilongjiang University, Harbin 150080) \\ ( ${ }^{b}$ Engineering Research Center of Pesticide of Heilongjiang University, Heilongjiang University, Harbin 150080)
}

\begin{abstract}
To obtain new herbicidal candidates with novel structure and high herbicidal activities, six new pyrazol compounds based on the structure of tembotrione were designed and synthesized. The meta substituent of benzene ring and the cyclohexadione ring in the original tembotrione structure are replaced. The six target compounds were achieved with 3-chloro-2-methylaniline as the starting material after nine steps reactions, namely diazotization, acetylization, oxidation, esterization, bromization, nucleophilic substitution, chlorination and condensation reactions. All the structures of target products and their intermediates are characterized and confirmed by ${ }^{1} \mathrm{H}$ NMR, ${ }^{13} \mathrm{C}$ NMR, FTIR and elemental analysis. Herbicidal activity tests are also conducted for the six compounds. The results show that each compound exhibits certain herbicidal activity toward mustard, chickweed, amur foxtail, chenopodium and polypogon fugax.

Keywords pyrazol; methylsulfonyl; tembotrione; synthesis; reaction conditions; herbicidal activities
\end{abstract}

随着世界农药的不断发展, 三酮类农药的应用越来 越广泛, 而具有新型结构的此类农药也已成为研究的热 点之一 ${ }^{[1 \sim 3]}$. 三酮类除草剂是 20 世纪 80 年代发现并开 发的一系列新型除草剂, 具有杀草谱广、用量低、对作 物安全, 人畜低毒, 易降解的优点, 具有广阔的市场前 景 ${ }^{[4]}$. 三酮类农药的主要结构特征体现为多羰基和甲磺 酰基官能团, 其很多衍生物均表现出广泛的除草活 性 $^{[5,6]}$.

20 世纪 90 年代欧洲的先正达公司首先报道了 2,4
位取代的苯环和环己二酮为主要结构的除草剂磺草 酮 ${ }^{[7]}$, 磺草酮被用于在玉米 $4 \sim 6$ 叶期喷雾防除玉米田大 多数一年生阔叶杂草及一部分禾本科杂草. 与此同时英 国 Zeneca 公司报道了与磺草酮结构类似的硝基磺草酮, 硝基基团取代磺草酮结构上 2 位的氯原子，硝基磺草酮 的杀草谱及用法也和磺草酮基本一致，但其除苍耳与豚 草的效果更好. 长期使用一种除草剂, 杂草会产生一定 的抗药性. 因此 2007 年拜耳公司研制并开发了磺草酮 结构上 3 位被三氟乙氧基甲基取代的新型除草剂环磺

*E-mail: yuyinghui@hlju.edu.cn

Received August 7, 2015; revised November 6, 2015; published online November 16, 2015.

Project supported by the the National Natural Science Foundation of China (No. 21371052) and the Heilongjiang Postdoctoral Scientific Research Foundation (No. LBH-Q14138).

国家自然科学基金(No. 21371052)和黑龙江省博士后科研启动基金(No. LBH-Q14138)资助项目. 
酮，其除草活性方面比硝磺酮和甲基磺草酮都要更高， 环磺酮的出现有效地解决了杂草抗性问题. 其主要活性 基团为苯环 3 位取代的三氟乙氧基甲基和环己二酮环官 能团. 本课题组针对环磺酮的结构, 并基于前期的一系 列研究, 保留其苯环上 2 位氯原子和 4 位甲磺酰基取代 基, 对苯环上的 3 位取代基团进行了修饰替换, 同时选 用具有良好生物活性的吡唑环, 取代原有结构上的环已 二酮环, 设计并合成出一系列的环磺酮类似物. 从结构 角度来说, 所合成的新化合物仍然保留环磺酮化合物的 主体结构, 且从修饰基团来看, 不同柔性基团、刚性基 团以及不同电子效应官能团的引入, 对其生物活性可能 产生不同影响 ${ }^{[8 \sim 10]}$.

本文基于如上设计思路, 对环磺酮结构中苯环的 3 位取代基及环已二酩环进行修饰替换，设计并合成了 6 个未经文献报道的新型化合物. 同时采用 ${ }^{1} \mathrm{H} N M R 、{ }^{13} \mathrm{C}$ NMR、FTIR、HPLC、元素分析和熔点测定等测试手段 对目标化合物及其中间体的结构进行了表征与确认. 此 外, 本文还对该 6 个化合物进行了除草活性测试, 测试 结果显示, 在剂量为 $150 \mathrm{~g}$ a.i./ha 时, 各化合物对对芥 菜、繁缕、看麦娘、小藜、棒头草均表现出良好的除草 效果. 目标化合物的合成路线如 Scheme 1 所示.

\section{1 结果与讨论}

\section{1 合成}

2-氯-6-甲硫基甲苯(2)是以 2-甲基-3-氯苯胺为起始 原料，经重氮化后与甲硫醇钠发生亲核反应制备得到 的. 此步反应盐酸的量尤为重要, 盐酸用量越少, 后处 理所用水量越少. 通过大量实验, 我们确定化合物的最 佳反应物料比为 $n_{1} / n_{\mathrm{HCl}}=1 / 4$.

2-氯-3-甲基-4-甲硫基苯乙酮(3) 是以二氯乙烷为溶 剂, 三氯化铝为催化剂, 乙酰氯为酰化试剂制备得到的. 在对反应溶剂进行选择的过程中，考虑到二氯乙烷的回 收率较高, 且价格适中, 确定其为最佳的反应溶剂.

2-氯-3-甲基-4-甲基磺酰基苯乙酮(4)的合成中以冰 醋酸与水为混合溶剂, 双氧水为氧化试剂 ${ }^{[11]}$. 此步反应 亚砜与砜同时生成, 待液相监测发现亚砜全部转化为砜 即可停止反应，反应的温度为 $80{ }^{\circ} \mathrm{C}$.

2-氯-3-甲基-4-甲基磺酰基苯甲酸(5)的合成是以水 为溶剂, 次氯酸钠为氧化试剂. 通过大量实验确定了化 合物的最佳反应物料比为 $n_{4} / n_{\mathrm{NaClO}}=1 / 6.3$.

2-氯-3-甲基-4-甲基磺酰基苯甲酸甲酯(6)的合成是 以甲醇为溶剂与酯化试剂, 二氯亚砜为催化剂. 甲醇既 作溶剂又作酯化试剂，降低了反应的成本.
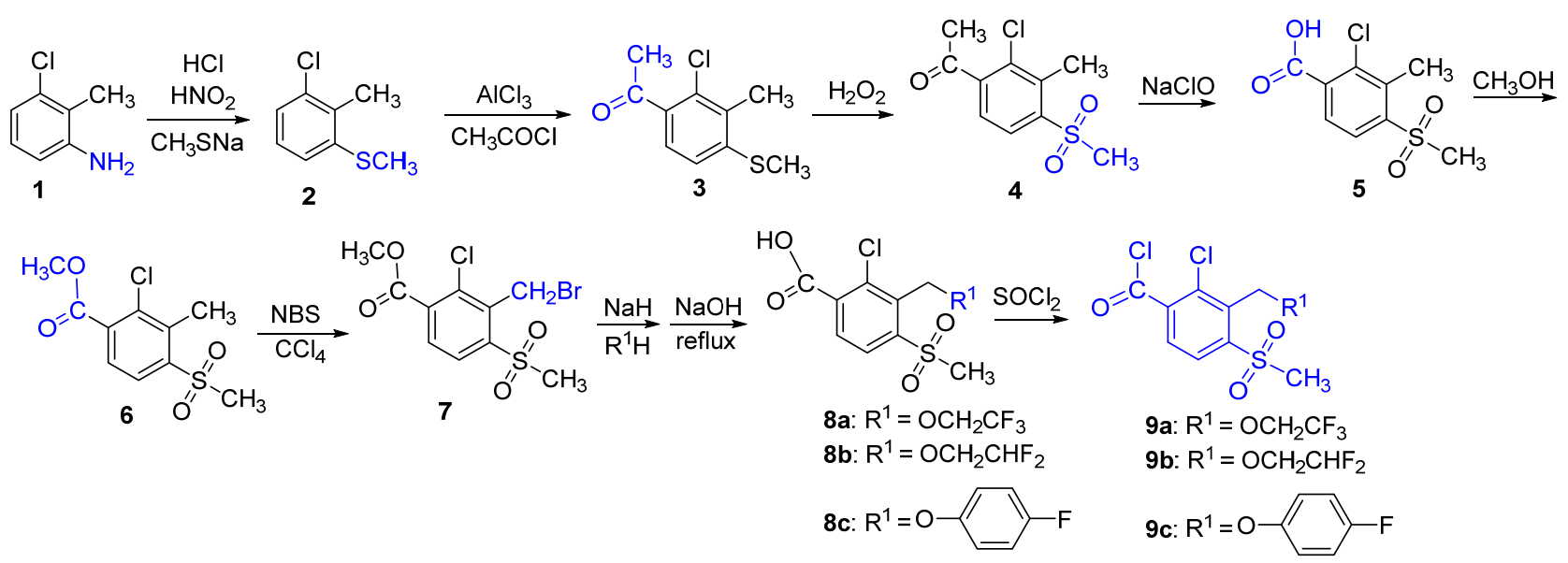
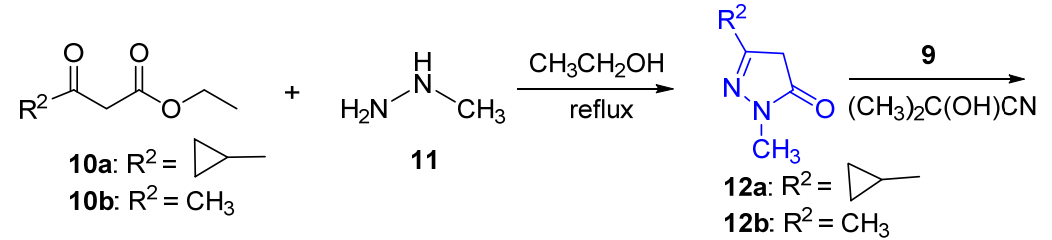

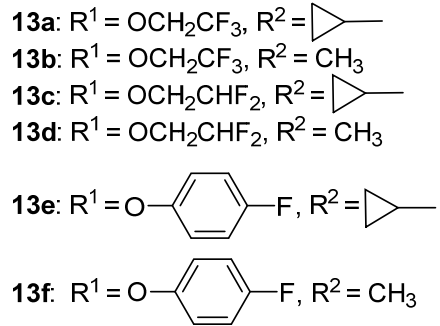

图式 1 目标化合物的合成路线

Scheme 1 Synthetic route of the target compounds 
3-溴甲基-2-氯-4-甲基磺酰基苯甲酸甲酯(7) ${ }^{[12]}$ 的合 成中以四氯化碳为溶剂, $N$-溴代丁二酰亚胺(NBS)为溴 化试剂. 此步反应中 NBS 的投加量尤为重要. 少量的 NBS 使原料反应不完全, 过量的 NBS 则会生成二溴取 代物. 通过大量实验, 最终确定化合物的最佳反应物料 比为 $n_{6} / n_{\mathrm{NBS}}=1 / 1.2$.

2-氯-3-(2,2,2-三氟乙氧基)甲基-4-甲基磺酰基苯甲 酸 $(8)$ 的合成中以四氢呋喃为溶剂, 氢化钠为碱, 与三氟 乙醇发生亲核反应, 液相追踪监测到原料消失后, 再加 入氢氧化钠升温回流水解即可.

2-氯-3-(2,2,2-三氟乙氧基)甲基-4-甲基磺酰基苯甲 酰氯(9)的合成中以二氯乙烷为溶剂, 二氯亚砜为酰化 试剂, DMF 为催化剂. 反应结束后蒸出二氯乙烷和少量 的二氯亚砜, 因酰氯极易水解, 加入无水二氯乙烷封闭 好待用.

1,3-二甲基-5-吡唑酮(12b) 是由乙酰乙酸乙酯与甲 基肼(40\%)关环得到 ${ }^{[13 \sim 16]}$, 由于酯羰基的活泼性没有羰 基的活泼性高, 且伯胺比仲胺的亲核性更强, 因此经过 脱水、再脱乙醇后获得产物. 同时在对反应溶剂进行选 择时, 发现产品在乙醇中析出较好, 因此确定其为反应 溶剂.

目标化合物 13a 是碱性条件下以二氯甲烷为溶剂, 三乙胺为碱, 丙酮氰醇为催化剂制备得到的 ${ }^{[17]}$, 三乙胺 为碱可提高反应速度, 降低反应温度至室温, 避免了许 多副反应的发生. 在酯重排过程中, 三乙胺提供碱性环 境, 丙酮氰醇为催化剂, 重排生成目标化合物.

\section{2 图谱分析}

以目标化合物 13d 为例对其结构表征图谱进行分 析. 从红外光谱数据上看在 $2970,1635,1590,1157,852$ $\mathrm{cm}^{-1}$ 附近有特征吸收峰. 目标化合物的 ${ }^{1} \mathrm{H}$ NMR 核磁共 振谱图中, 吡唑环与双键相连的甲基受双键的影响向低 场移动, 在 $\delta 2.16$ 处出现单峰; 吡唑环上与氮相连的甲 基受相连氮原子的影响向低场移动; 甲砜基上的甲基氢 受砜基的吸电子效应影响向低场移动, 并与吡唑环上与 氮相连的甲基氢重合, 在 $\delta 3.35$ 处出现单峰; 与苯环相 连的亚甲基氢受烷氧基吸电子效应的影响向低场移动, 在 $\delta 5.17$ 处出现单峰; 化学位移 $\delta 6.21,3.88$ 处出现的多 重峰为二氟乙醇上氢原子的特征峰; 在 $\delta 8.03,7.57$ 出现 多重峰为苯环上的 2 个 H. ${ }^{13} \mathrm{C}$ NMR 核磁共振谱图中, 脂肪碳 $\delta 8.47,9.10,2599,45.48,62.55$; 芳环碳 $\delta 103.96$, 112.67, 115.04, 117.42, 128.69, 133.67, 141.92, 147.35, 186.11. 元素分析 Anal. calcd for $\mathrm{C}_{16} \mathrm{H}_{17} \mathrm{~N}_{2}$ : C $45.45, \mathrm{H}$ 4.05, N 6.63; found C 45.77, H 4.24, N 6.61 说明产品纯 度高. 高分辨质谱 $\mathrm{HRMS}$ calcd for $\mathrm{C}_{16} \mathrm{H}_{17} \mathrm{ClF}_{2} \mathrm{~N}_{2} \mathrm{O}_{5} \mathrm{~S}$ $445.0500[\mathrm{M}+\mathrm{Na}]^{+}, 867.1010[2 \mathrm{M}+\mathrm{Na}]^{+}$; found
$445.0406[\mathrm{M}+\mathrm{Na}]^{+}, 867.0938[2 \mathrm{M}+\mathrm{Na}]^{+}$说明分子量正 确. 综合 FTIR、 ${ }^{1} \mathrm{H}$ NMR、 ${ }^{13} \mathrm{C}$ NMR、元素分析、HRMS 等数据分析, 充分证实了目标化合物的结构.

化合物 13a, 13b, 13c, 13e, 13f 的分子结构与 13d 相 似, 此处不作详细介绍.

\section{3 除草活性}

参照创制农药生物活性评价 SOP(除草卷)标准, 使 用活体盆栽法, 以除草剂环磺酮为参照物, 将本文合成 的目标化合物 $11 \mathrm{a} \sim 11 \mathrm{f}$ 对芥菜、繁缕、看麦娘、小莍、 棒头草五种杂草的防除效果进行普筛测试, 测试结果见 表 1 .

表 1 目标化合物的除草活性测试结果 ${ }^{a}$

Table 1 Herbicidal activity test results of the target compounds

\begin{tabular}{cccccc}
\hline Compd. & 芥菜 & 繁缕 & 看麦娘 & 小藜 & 棒头草 \\
\hline 11a & 100 & 100 & 80 & 80 & 95 \\
$\mathbf{1 1 b}$ & 100 & 100 & 20 & 80 & 20 \\
$\mathbf{1 1 c}$ & 100 & 100 & 100 & 100 & 100 \\
$\mathbf{1 1 d}$ & 100 & 100 & 95 & 100 & 100 \\
$\mathbf{1 1 e}$ & 20 & 50 & 40 & 90 & 100 \\
$\mathbf{1 1 f}$ & 20 & 100 & 95 & 85 & 100 \\
环磺酮 & 100 & 100 & 100 & 100 & 100 \\
\hline
\end{tabular}

${ }^{a}$ 图中数字为百分数, 特指对对应杂草的防除 $(\%)$; 测试剂量为 $150 \mathrm{~g}$ a.i./ha.

除草活性实验结果表明, 在 $150 \mathrm{~g}$ a.i./ha 时不同化 合物显示出不同的除草活性, 11a 11f 均对杂草有一定 的防除效果，其中 11a 对杂草的防除率达到 $80 \%$ 以上， $11 \mathrm{c}$ 和 11d 对杂草的防除率达到 95\%以上, 与商品化的 环磺酮的除草效率相当, 因此实现了对环磺酮结构进行 改进和修饰以消除长期使用单一结构除草剂产生抗药 性的目的, 为今后更深入研究提供了有益的数据支持.

\section{2 结论}

本文以为 2-甲基-3-氯苯胺起始原料, 经过重氮化、 乙酰基化、双氧水氧化、次氯酸钠氧化、酯化、溴化、 亲核反应、水解反应最终合成了主要中间体 2-氯(-3-( $\left.\mathrm{R}^{1}\right)$ 甲基-4-甲基磺酰基苯甲酸, 并参照相关文献对每一步 反应进行了优化和改进. 以上述甲磺酰基苯甲酸为原料 合成其酰氯, 然后酰氯与吡唑酮成酯, 最终在丙酮氧醇 催化下重排转位得到了 6 个未被文献报道的化合物，通 过该合成路线获得的产品纯度较好, 收率较高, 反应条 件温和，后处理简便，可操作性强，适合规模化生产. 本文同时研究了溶剂、反应温度、反应时间、物料配比、 催化剂等反应条件及因素对各步单元反应的影响, 确定 了各步反应的最佳条件，获得了目标化合物及其中间体 的分析测试条件. 采用 ${ }^{1} \mathrm{H}$ NMR、 ${ }^{13} \mathrm{C}$ NMR、FTIR、 HPLC、元素分析和熔点测定等测试手段对目标化合物 
及其中间体的结构进行了表征与确认. 6 个目标化合物 除草活性测试实验结果表明, 在剂量为 $150 \mathrm{~g}$ a.i./ha 时, 各化合物对对芥菜、繁缕、看麦娘、小藜、棒头草均表 现出较好的除草效果, 为新农药除草剂的开发提供了理 论支持.

\section{3 实验部分}

\section{1 仪器与试剂}

MPA100 全自动熔点仪(温度计未校正); Bruker-400 型核磁共振仪 (以 $\mathrm{CDCl}_{3}$ 或 DMSO- $d_{6}$ 作溶剂, 以 $\mathrm{TMS}$ 为 内标); 红外光谱仪 FTIR-8400S; Yanaco MT-3CHN 元素 分析仪; 高压液相色谱仪 LC-20A. 主要原料乙酰乙酸 乙酯、甲基肼、3-氯-2-甲基苯胺为工业品，其余试剂均 为化学纯或试剂纯.

各化合物纯度均由高效液相色谱仪测试数据为依 据，液相测试方法：面积归一化法; 液相色谱分析条件: $\mathrm{C}_{18}$ 反相硅胶柱, 波长 $230 \mathrm{~nm}$, 流动相为 $V$ (甲醇) : $V$ (磷 酸水, $0.5 \%$ 磷酸 $)=80: 20$, 流速 $0.8 \mathrm{~mL} / \mathrm{min}$.

\section{2 化合物 $8 \mathrm{a} \sim 8 \mathrm{c}$ 的合成}

\subsubsection{2-氯-6-甲硫基甲苯的(2)的合成}

在连接有回流冷凝装置的 $2 \mathrm{~L}$ 四口瓶中, 加入原料 2-甲基-3-氯苯胺 $200.2 \mathrm{~g}(1.42 \mathrm{~mol})$, 水 $380.0 \mathrm{~g}$ (21.11 $\mathrm{mol})$, 温度不超过 $15{ }^{\circ} \mathrm{C}$ 下滴加浓盐酸 $417.1 \mathrm{~g}$ (4.00 $\mathrm{mol})$, 随着盐酸的加入有白色固体析出, 滴加完盐酸继 续摚拌 $20 \mathrm{~min}$. 冷却降温至 $5{ }^{\circ} \mathrm{C}$, 滴加亚硝酸钠水溶液 $\left(\mathrm{NaNO}_{2}: 102.0 \mathrm{~g}, \mathrm{H}_{2} \mathrm{O}: 290.0 \mathrm{~g}\right)$, 保持反应温度不超过 $10{ }^{\circ} \mathrm{C}$, 随着反应的进行, 白色固体溶解, 溶液变为红棕 色，滴加完毕后，继续摚拌 $30 \mathrm{~min}$, 溶液待用.

在连接有冷凝回流装置的 $2 \mathrm{~L}$ 四口瓶中, 先加入 $20 \%$ 甲硫醇钠 $595.0 \mathrm{~g}(1.70 \mathrm{~mol})$, 氢氧化钠 $56.0 \mathrm{~g}(1.40$ $\mathrm{mol})$, 碳酸钾 $62.1 \mathrm{~g}(0.45 \mathrm{~mol})$, 搅拌至固体完全溶解, 向其中滴加上步待用溶液, 温度控制不超过 $10{ }^{\circ} \mathrm{C}$, 滴 加完毕后搅拌过夜. 加入 $300 \mathrm{~mL}$ 二氯乙烷萃取, 减压 蒸出二氯乙烷, 精馏得黄色溶液 $\mathbf{2}^{[18]} 180.1 \mathrm{~g}$. 收率 $75.1 \%$, 纯度 99.5\%. ${ }^{1} \mathrm{H}$ NMR (400 $\left.\mathrm{MHz}, \mathrm{CDCl}_{3}\right) \delta: 7.21$ (dd, $J=7.7,1.5 \mathrm{~Hz}, 1 \mathrm{H}), 7.13(\mathrm{t}, J=7.8 \mathrm{~Hz}, 1 \mathrm{H}), 7.11 \sim$ 7.07 (m, 1H), 2.49 (s, 3H), 2.47 (s, 3H).

\subsubsection{2-氯-3-甲基-4-甲硫基苯乙酮(3)的合成}

在连接有回流装置的 $500 \mathrm{~mL}$ 四口瓶中, 加入二氯 乙烷 $200 \mathrm{~mL}, \mathrm{AlCl}_{3} 46.6 \mathrm{~g}(0.35 \mathrm{~mol})$, 滴加乙酰氯 $27.5 \mathrm{~g}$ (0.35 mol), 温度控制不超过 $20{ }^{\circ} \mathrm{C}$, 溶液形成黄色悬浮 液. 向其中滴入 2-氯-6-甲硫基甲苯(2) $51.6 \mathrm{~g} \mathrm{(0.30} \mathrm{mol)}$ 在 $200 \mathrm{~mL}$ 二氯乙烷的溶液, 温度控制不超过 $20{ }^{\circ} \mathrm{C}$, 摚 拌过夜, 反应物倒入冰水和浓盐酸的混合液中. 经二氯
乙烷萃取, 水洗后浓缩, 得到黑色液体 $\mathbf{3}^{[18]} 62.8 \mathrm{~g}$. 收率 99.1\%, 纯度 $97.1 \%$. ${ }^{1} \mathrm{H}$ NMR (400 $\left.\mathrm{MHz}, \mathrm{CDCl}_{3}\right) \delta: 7.36$ (s, 1H), $7.08(\mathrm{~s}, 1 \mathrm{H}), 3.74(\mathrm{~s}, 3 \mathrm{H}), 2.63(\mathrm{~s}, 3 \mathrm{H}), 2.44 \sim 2.43$ $(\mathrm{m}, 3 \mathrm{H})$.

\subsubsection{2-氯-3-甲基-4-甲基磺酰基苯乙酮(4)的合成}

在连接有回流装置的 $500 \mathrm{~mL}$ 四口瓶中加入 2-氯-3甲基-4-甲硫基苯乙酩(3) $64.2 \mathrm{~g}(0.30 \mathrm{~mol})$, 冰醋酸 300 $\mathrm{mL}$, 铇酸钠 $1.0 \mathrm{~g}$. 向其中滴入 $30 \%$ 过氧化氢 $70.3 \mathrm{~g}(0.62$ $\mathrm{mol})$, 升温到 $80{ }^{\circ} \mathrm{C}$ 并保温 $1 \mathrm{~h}$ 后用水稀释，抽滤获得固 体产物，将产物用水洗后干燥. 获得白色固体 $468.6 \mathrm{~g}$, 收率 $93.2 \%$, 纯度 $97.0 \%$. m.p. 110.6 $111.1{ }^{\circ} \mathrm{C}$ (文献 值 ${ }^{[18]} 110.0 \sim 111.0{ }^{\circ} \mathrm{C}$ ); ${ }^{1} \mathrm{H}$ NMR (400 MHz, DMSO- $d_{6}$ ) $\delta: 8.00(\mathrm{~d}, J=8.2 \mathrm{~Hz}, 1 \mathrm{H}), 7.65$ (d, $J=8.2 \mathrm{~Hz}, 1 \mathrm{H}), 3.31$ (s, 3H), 2.72 (s, 3H), 2.58 (s, 3H).

\subsubsection{2-氯-3-甲基-4-甲基磺酰基苯甲酸 (5)的合成}

在连接有回流装置的 $1 \mathrm{~L}$ 四口瓶中, 加入 $300 \mathrm{~mL}$ 二氧六环，2-氯-3-甲基-4-甲基磺酰基苯乙酮(4) $24.6 \mathrm{~g}$ $(0.10 \mathrm{~mol})$, 向其中滴加 $10 \%$ 次氯酸钠 $468.7 \mathrm{~g}(0.63 \mathrm{~mol})$, 加热到 $80{ }^{\circ} \mathrm{C}$, 保温 $2 \mathrm{~h}$. 待液相检测原料完全消失后, 减压蒸出大部分二氧六环, 降温到 $40{ }^{\circ} \mathrm{C}$, 滴加盐酸到 $\mathrm{pH}=2$, 此时析出大量白色固体, 搅拌冷却到室温, 过 滤. $50{ }^{\circ} \mathrm{C}$ 烘干得到白色固体 $523.6 \mathrm{~g}$, 收率 $95.0 \%$, 纯度 $98.6 \%$. m.p. $230.5 \sim 231.6{ }^{\circ} \mathrm{C}$ (文献值 ${ }^{[18]} 230.0 \sim$ $\left.231.0{ }^{\circ} \mathrm{C}\right) ;{ }^{1} \mathrm{H}$ NMR $\left(400 \mathrm{MHz}\right.$, DMSO- $\left.d_{6}\right) \delta: 7.98(\mathrm{~d}, J=$ $8.2 \mathrm{~Hz}, 1 \mathrm{H}), 7.72$ (d, $J=8.2 \mathrm{~Hz}, 1 \mathrm{H}), 3.31$ (s, 3H), 2.72 (s, $3 \mathrm{H})$.

3.2.5 2-氯-3-甲基-4-甲基磺酰基苯甲酸甲酯(6)的合 成

在连接有回流装置的 $500 \mathrm{~mL}$ 四口瓶中，加入 200 $\mathrm{mL}$ 甲醇, 搅拌下加入 2-氯-3-甲基-4-甲基磺酰基苯甲酸 (5) $24.8 \mathrm{~g}(0.10 \mathrm{~mol})$, 滴加二氯亚砜 $5.9 \mathrm{~g}(0.05 \mathrm{~mol})$, 控 温不超过 $25{ }^{\circ} \mathrm{C}$, 滴加完搅拌 $10 \mathrm{~min}$, 升温回流 $2 \mathrm{~h}$. 冷 却降温，抽滤，烘干后得固体 $623.4 \mathrm{~g}$, 收率 $93.4 \%$, 纯 度 98.0\%. m.p. 107.6 108.5 ${ }^{\circ} \mathrm{C}$ (文献值 ${ }^{[18]} 107.0 \sim$ $108.0{ }^{\circ} \mathrm{C} ;{ }^{1} \mathrm{H}$ NMR $\left(400 \mathrm{MHz}\right.$, DMSO- $\left.d_{6}\right) \delta: 8.01(\mathrm{~d}, J=$ $8.2 \mathrm{~Hz}, 1 \mathrm{H}), 7.78(\mathrm{~d}, J=8.2 \mathrm{~Hz}, 1 \mathrm{H}), 3.90$ (s, $3 \mathrm{H}), 3.32$ (s,3H), $2.73(\mathrm{~s}, 3 \mathrm{H})$.

3.2.6 3-溴甲基-2-氯-4-甲基磺酰基苯甲酸甲酯(7)的 合成

在连接有回流装置的 $500 \mathrm{~mL}$ 四口瓶中, 加入 400 $\mathrm{mL} \mathrm{CCl}_{4}$, 搅拌下加入 2-氯-3-甲基-4-甲基磺酰基苯甲酸 甲酯(6) $52.4 \mathrm{~g}(0.20 \mathrm{~mol})$ 和偶氮二异丁腈 $0.2 \mathrm{~g}$, 然后分 5 次加入 NBS $37.4 \mathrm{~g}(0.21 \mathrm{~mol})$, 升温回流 $8 \mathrm{~h}$. 降温抽滤 除去固体后, 将剩余液体蒸出四氯化碳, 加入 $100 \mathrm{~mL}$ 乙醚，搅拌 $10 \mathrm{~min}$ 抽滤, $50{ }^{\circ} \mathrm{C}$ 烘干获得黄色固体 751.1 
$\mathrm{g}$, 收率 75.2\%, 纯度 95.5\%. m.p. 74.6 75.4 ${ }^{\circ} \mathrm{C}$ (文献 值 $\left.{ }^{[18]} 74.0 \sim 75.0{ }^{\circ} \mathrm{C}\right) ;{ }^{1} \mathrm{H}$ NMR (400 MHz, DMSO- $\left.d_{6}\right) \delta$ : 8.09 (d, $J=8.3 \mathrm{~Hz}, 1 \mathrm{H}), 7.96$ (d, $J=8.3 \mathrm{~Hz}, 1 \mathrm{H}), 5.17$ (s, $2 \mathrm{H}), 3.93$ (s, 3H), 3.42 (s, 3H).

3.2.7 2-氯-3-(2,2,2-三氟乙氧基)甲基-4-甲基磺酰基 苯甲酸 $(8 \mathbf{a})$ 的合成

在连接有回流装置的 $500 \mathrm{~mL}$ 四口瓶中, 加入 200 $\mathrm{mL}$ THF, 摚拌下加入 $\mathrm{NaH} 5.2 \mathrm{~g}(0.12 \mathrm{~mol})$, 温度不超过 $20{ }^{\circ} \mathrm{C}$, 滴加三氟乙醇 $12.0 \mathrm{~g}(0.12 \mathrm{~mol})$, 滴加完搅拌 30 $\mathrm{min}$, 加入 3 -溴甲基-2-氯-4-甲基磺酰基苯甲酸甲酯(7) $34.0 \mathrm{~g}(0.10 \mathrm{~mol})$, 室温反应 $4 \mathrm{~h}$, 加入氢氧化钠 $4.8 \mathrm{~g}$ $(0.12 \mathrm{~mol})$, 水 $200 \mathrm{~mL}$, 升温回流 $2 \mathrm{~h}$. 冷却到 $20{ }^{\circ} \mathrm{C}$, 加 $\mathrm{HCl}$ 至 $\mathrm{pH}=2$. 抽滤, $50{ }^{\circ} \mathrm{C}$ 烘干得到黄色固体 8a, 收率 95.0\%, 纯度 $97.2 \%$. m.p 154.8 155.8 ${ }^{\circ} \mathrm{C} ;{ }^{1} \mathrm{H}$ NMR(400 MHz, DMSO- $\left.d_{6}\right) \delta: 8.31 \sim 8.23(\mathrm{~m}, 2 \mathrm{H}), 8.12(\mathrm{~d}, J=8.2$ $\mathrm{Hz}, 1 \mathrm{H}), 8.00(\mathrm{~d}, J=8.2 \mathrm{~Hz}, 1 \mathrm{H}), 7.41 \sim 7.26(\mathrm{~m}, 2 \mathrm{H})$, $5.71(\mathrm{~s}, 2 \mathrm{H}), 3.36(\mathrm{~s}, 3 \mathrm{H})$.

化合物 $8 \mathrm{~b}$ 和 $8 \mathrm{c}$ 合成方法同化合物 8a.

2-氯-3-(2,2-二氟乙氧基)甲基-4-甲基磺酰基苯甲酸 (8b): 黄色固体, 收率 93.2\%, 纯度 99.4\%. m.p 138.0 $139.0{ }^{\circ} \mathrm{C} ;{ }^{1} \mathrm{H}$ NMR (400 MHz, DMSO- $\left.d_{6}\right) \delta: 8.18$ (d, $J=$ $8.3 \mathrm{~Hz}, 1 \mathrm{H}), 8.01(\mathrm{~d}, J=8.3 \mathrm{~Hz}, 1 \mathrm{H}), 5.94(\mathrm{~s}, 1 \mathrm{H}), 5.35(\mathrm{~s}$, $2 \mathrm{H}), 3.90$ (td, $J=14.1,3.8 \mathrm{~Hz}, 2 \mathrm{H}), 3.26$ (s, 3H).

2-氯-3-(4-氟苯氧基)甲基-4-甲基磺酰基苯甲酸(8c): 黄色固体, 收率 $96.1 \%$, 纯度 $99.4 \%$. m.p 208.3 $209.6{ }^{\circ} \mathrm{C} ;{ }^{1} \mathrm{H}$ NMR(400 MHz, DMSO- $\left.d_{6}\right) \delta: 8.11$ (d, $J=$ $8.2 \mathrm{~Hz}, 1 \mathrm{H}), 7.97(\mathrm{~d}, J=8.2 \mathrm{~Hz}, 1 \mathrm{H}), 7.25 \sim 6.98(\mathrm{~m}, 4 \mathrm{H})$, $5.55(\mathrm{~s}, 2 \mathrm{H}), 3.34(\mathrm{~s}, 3 \mathrm{H})$.

\section{3 目标化合物 13a 13f 的合成}

\section{3 .1 化合物 $9 \mathbf{a} \sim 9 \mathbf{c}$ 的合成}

在连接有回流装置的 $250 \mathrm{~mL}$ 四口瓶中, 加入 200 $\mathrm{mL}$ 二氯乙烷, 搅拌下加入 2 -氯-3-(2,2,2-三氟乙氧基)甲 基-4-甲基磺酰基苯甲酸 $(8 \mathrm{a}) 6.7 \mathrm{~g}(0.02 \mathrm{~mol})$, 滴加二氯 亚砜 $2.9 \mathrm{~g}(0.02 \mathrm{~mol})$, 温度控制不超过 $20{ }^{\circ} \mathrm{C}$, 滴加完毕 后升温回流 $1 \mathrm{~h}$, 反应完毕后减压蒸出二氯乙烷和少量 的二氯亚砜. 加入 $50 \mathrm{~mL}$ 二氯乙烷溶液, 封闭好待用.

\subsection{2 化合物 $\mathbf{1 2 a} \sim \mathbf{1 2 b}$ 的合成}

在连接有回流装置的 $250 \mathrm{~mL}$ 四口瓶中, 加入乙酰 乙酸乙酯 $195.0 \mathrm{~g}(1.50 \mathrm{~mol})$, 滴加 $172.5 \mathrm{~g}(1.50 \mathrm{~mol})$ 质 量浓度为 $40 \%$ 的甲基肼, 通过冷水浴控温在 $16{ }^{\circ} \mathrm{C}$ 的条 件下反应 $2 \mathrm{~h}$, 减压蒸去生成的乙醇, 向反应体系中加 入甲苯常压回流分水, 冷却降温析出浅黄色固体 $\mathbf{1 2 b}$, 固体经过滤和干燥后得 $159.9 \mathrm{~g}$, 收率 $95.2 \%$, 纯度 99.8\%. m.p. $117.1 \sim 117.5{ }^{\circ} \mathrm{C}$ (文献值 $\left.{ }^{[13]} 117.0{ }^{\circ} \mathrm{C}\right) ;{ }^{1} \mathrm{H}$ NMR (400 MHz, DMSO- $\left.d_{6}\right) \delta: 2.07$ (s, 3H), 3.15 (s, 2H), $3.24(\mathrm{~s}, 3 \mathrm{H})$.

\subsection{3 目标化合物 $\mathbf{1 3 a} \sim \mathbf{1 3 f}$ 的合成}

在连接有回流装置的 $250 \mathrm{~mL}$ 四口瓶中, 加入 200 $\mathrm{mL}$ 二氯乙烷, 搅拌下加入 1,3-二甲基-5-吡唑酮 (12b) $2.3 \mathrm{~g}(0.02 \mathrm{~mol})$, 滴加三乙胺 $6.0 \mathrm{~g}(0.06 \mathrm{~mol})$, 温度控制 不超过 $20{ }^{\circ} \mathrm{C}$, 滴加上述第 3.3.1 节中待用溶液 $9 a$, 温度 控制不超过 $35{ }^{\circ} \mathrm{C}$, 液相监测至反应结束. 滴加丙酮氰 醇 $0.5 \mathrm{~mL}$, 搅拌反应过夜. 加入水 $100 \mathrm{~mL}$, 加 $\mathrm{HCl}$ 至 $\mathrm{pH}$ $=2$, 分液, 水洗, 减压蒸出二氯乙烷, 加入甲醇 $80 \mathrm{~mL}$, 摚拌析出固体, 抽滤, $40{ }^{\circ} \mathrm{C}$ 烘干得目标化合物 $\{[2$-氯 -3-(2,2,2-三氟乙氧基)甲基-4-甲基磺酰基]-苯基 $\}$-[(1,3二甲基-5-羟基)吡唑-4]-酮(13b), 白色絮状固体, 纯度 95.8\%, 收率 94.3\%. m.p. 130.3 131.1 ${ }^{\circ} \mathrm{C} ;{ }^{1} \mathrm{H}$ NMR (400 $\left.\mathrm{MHz}, \mathrm{CDCl}_{3}\right) \delta: 8.14(\mathrm{~d}, J=8.1 \mathrm{~Hz}, 1 \mathrm{H}), 7.33$ (d, $J=8.1$ $\mathrm{Hz}, 1 \mathrm{H}), 3.73$ (s, 2H), 3.64 (s, 2H), 3.14 (s, 3H), 2.84 (s, $3 \mathrm{H}), 1.71(\mathrm{~s}, 3 \mathrm{H}) ;{ }^{13} \mathrm{C}$ NMR (100 MHz, DMSO- $\left.d_{6}\right) \delta$ : $160.66,146.33,145.16,144.13,136.22,135.02,134.90$, $132.37,128.83,94.27,68.00,67.67,66.31,45.23,34.87$, 14.51; IR (KBr) $v: 2918,1653,1538,1141,849,750,638$ $\mathrm{cm}^{-1}$; Anal. calcd for $\mathrm{C}_{16} \mathrm{H}_{16} \mathrm{~N}_{2}$ : C 43.59, H 3.66, N 6.35; found C 43.68, H 3.76, N 6.31.

化合物 13a, 13c 13f 合成方法同化合物 13b.

$\{[2-$ 氯-3-(2,2,2-三氟乙氧基)甲基-4-甲基磺酰基 $]$ 苯基\}-[(1-甲基-3-环丙基-5-羟基)吡唑-4]-酮(13a): 淡黄 色粉末固体，纯度 $96.2 \%$, 收率 93.4\%. m.p. 75.8 $76.8{ }^{\circ} \mathrm{C} ;{ }^{1} \mathrm{H}$ NMR (400 MHz, DMSO- $\left.d_{6}\right) \delta: 8.03(\mathrm{~d}, J=$ $8.1 \mathrm{~Hz}, 1 \mathrm{H}), 7.64(\mathrm{~d}, J=7.6 \mathrm{~Hz}, 1 \mathrm{H}), 5.23(\mathrm{~s}, 2 \mathrm{H}), 4.27$ (d, $J=9.4 \mathrm{~Hz}, 2 \mathrm{H}), 3.33(\mathrm{t}, J=5.4 \mathrm{~Hz}, 6 \mathrm{H}), 0.77(\mathrm{~s}, 4 \mathrm{H}) ;{ }^{13} \mathrm{C}$ NMR $\left(100 \mathrm{MHz}\right.$, DMSO- $\left.d_{6}\right) \delta: 186.04,147.35,141.93$, $133.52,128.86,128.73,126.23,123.45,103.94,67.93$, 67.60, 66.32, 62.53, 45.40, 25.98, 9.08, 8.42; IR (KBr) $v$ : 2927, 1653, 1525, 1146, 867, 761, $668 \mathrm{~cm}^{-1}$. Anal. calcd for $\mathrm{C}_{18} \mathrm{H}_{18} \mathrm{~N}_{2}$ : C 46.31, H 3.89, N 6.00; found C 46.24, H 4.09, N 6.07 .

$\{$ [2-氯-3-(2,2-二氟乙氧基)甲基-4-甲基磺酰基 $]$-苯 基\}-[(1-甲基-3-环丙基-5-羟基)吡唑-4]-酮(13c): 黄色粉 末固体, 纯度 $98.4 \%$, 收率 95.6\%. m.p. 94.7 95.5 ${ }^{\circ} \mathrm{C}$; ${ }^{1} \mathrm{H}$ NMR (400 MHz, DMSO- $\left.d_{6}\right) \delta: 8.02(\mathrm{~d}, J=8.1 \mathrm{~Hz}$, $1 \mathrm{H}), 7.63(\mathrm{~d}, J=8.0 \mathrm{~Hz}, 1 \mathrm{H}), 6.36 \sim 6.05(\mathrm{~m}, 1 \mathrm{H}), 5.16(\mathrm{~s}$, $2 \mathrm{H}), 3.84(\mathrm{~m}, 2 \mathrm{H}), 3.35(\mathrm{~s}, 6 \mathrm{H}), 0.77(\mathrm{~s}, 4 \mathrm{H}) ;{ }^{13} \mathrm{C} \mathrm{NMR}$ $\left(100 \mathrm{MHz}, \mathrm{DMSO}-d_{6}\right) \delta: 186.11,147.36,141.92,133.67$, $128.69,117.42,115.04,112.67,103.96,62.55,45.48$, 25.99, 9.10, 8.47; IR (KBr) v: 2929, 1653, 1521, 1198, $860,668,569 \mathrm{~cm}^{-1}$. Anal. calcd for $\mathrm{C}_{18} \mathrm{H}_{19} \mathrm{~N}_{2}: \mathrm{C} 48.16, \mathrm{H}$ 4.27, N 6.24; found C 47.71, H 4.21, N 6.24. 
$\{[2$-氯-3-(2,2-二氟乙氧基)甲基-4-甲基磺酰基]-苯 基 $\}$-[(1,3-二甲基-5-差基)吡唑-4]-酮(13d): 淡黄色粉末 固体，纯度 $97.5 \%$, 收率 $96.2 \%$. m.p. $147.5 \sim 148.2{ }^{\circ} \mathrm{C}$; ${ }^{1} \mathrm{H}$ NMR $\left(400 \mathrm{MHz}\right.$, DMSO- $\left.d_{6}\right) \delta: 8.03(\mathrm{~d}, J=8.1 \mathrm{~Hz}$, $1 \mathrm{H}), 7.57(\mathrm{~d}, J=8.1 \mathrm{~Hz}, 1 \mathrm{H}), 6.21(\mathrm{~s}, 1 \mathrm{H}), 5.17(\mathrm{~s}, 2 \mathrm{H})$, $3.88(\mathrm{td}, J=15.4,3.4 \mathrm{~Hz}, 2 \mathrm{H}), 3.35$ (s, 6H), 2.16 (s, 3H); ${ }^{13} \mathrm{C}$ NMR $\left(100 \mathrm{MHz}\right.$, DMSO- $\left.d_{6}\right) \delta: 186.03,148.40,147.25$, $141.91,133.56,128.75,128.61,117.42,115.04,112.67$, 103.47, 69.78, 65.91, 45.47, 14.58; IR (KBr) v: 2927, 1653, 1539, 1208, 862, 668, $567 \mathrm{~cm}^{-1}$. Anal. calcd for $\mathrm{C}_{16} \mathrm{H}_{17} \mathrm{~N}_{2}$ : C 45.45, H 4.05, N 6.63; found C 45.77, H 4.24, $\mathrm{N}$ 6.61. HRMS calcd for $\mathrm{C}_{16} \mathrm{H}_{17} \mathrm{ClF}_{2} \mathrm{~N}_{2} \mathrm{O}_{5} \mathrm{~S}[\mathrm{M}+\mathrm{Na}]^{+}$ 445.0500, $[2 \mathrm{M}+\mathrm{Na}]^{+} 867.1010$, found $[\mathrm{M}+\mathrm{Na}]$ 445.0406, $[2 \mathrm{M}+\mathrm{Na}]^{+}$867.0938.

\{[2-氯-3-(4-氟苯氧基)甲基-4-甲基磺酰基 $]$-苯 基 -[(1-甲基-3-环丙基-5-差基)吡唑-4]-酮(13e): 黄色粉 末固体, 纯度 $97.1 \%$, 收率 $95.4 \%$, m.p. $174.8 \sim 175.8{ }^{\circ} \mathrm{C}$; ${ }^{1} \mathrm{H}$ NMR $\left(400 \mathrm{MHz}\right.$, DMSO- $\left.d_{6}\right) \delta: 8.08(\mathrm{~d}, J=8.1 \mathrm{~Hz}$, 1H), 7.69 (d, $J=7.8 \mathrm{~Hz}, 1 \mathrm{H}), 7.22 \sim 7.08(\mathrm{~m}, 4 \mathrm{H}), 5.55$ (s, 2H), 3.35 (d, $J=15.7 \mathrm{~Hz}, 6 \mathrm{H}), 0.79$ (s, 4H); ${ }^{13} \mathrm{C}$ NMR $(100$ $\mathrm{MHz}$, DMSO- $\left.d_{6}\right) \delta: 186.02,158.64,156.29,155.02$, $147.41,142.09,133.13,128.88,116.66,116.43,116.33$, 103.94, 63.76, 45.50, 9.10, 8.44; IR (KBr) v: 2931, 1653, $1539,1204,826,755,546 \mathrm{~cm}^{-1}$. Anal. calcd for $\mathrm{C}_{22} \mathrm{H}_{20} \mathrm{~N}_{2}$ : C 55.17, H 4.21, N 5.85; found C 55.47, H 4.31, N 5.95.

$\{$ [2-氯-3-(4-氟苯氧基)甲基-4-甲基磺酰基 $]$-苯 基 $\}$-[(1,3-二甲基-5-差基)吡唑-4]-酮(13f)：黄色粉末固 体，纯度 99.4\%, 收率 90.6\%. m.p. 163.9 164.5 ${ }^{\circ} \mathrm{C} ;{ }^{1} \mathrm{H}$ NMR (400 MHz, DMSO- $\left.d_{6}\right) \delta: 8.07(\mathrm{~d}, J=8.1 \mathrm{~Hz}, 1 \mathrm{H})$, $7.63(\mathrm{~d}, J=8.1 \mathrm{~Hz}, 1 \mathrm{H}), 7.24 \sim 7.06(\mathrm{~m}, 4 \mathrm{H}), 5.55(\mathrm{~s}, 2 \mathrm{H})$, 3.35 (s, 3H), 3.34 (s, 3H), 2.18 (s, 3H); ${ }^{13} \mathrm{C}$ NMR (100 $\left.\mathrm{MHz}, \mathrm{DMSO}-d_{6}\right) \quad \delta: 185.95,158.68,156.33,155.03$, $147.38,142.09,133.13,128.89,116.68,116.45,116.38$, 103.49, 63.80, 45.53, 14.60; IR (KBr) v: 2928, 1653, 1539, 1206, 830, 736, $558 \mathrm{~cm}^{-1}$. Anal. calcd for $\mathrm{C}_{20} \mathrm{H}_{18} \mathrm{~N}_{2}$ : C 55.04, H 4.01, N 6.19; found C 55.44, H 4.21, N 6.39.

致谢 本文中的除草活性测试委托浙化院生测安评中 心的唐伟老师等人完成, 特此致谢.

辅助材料(Supporting Information) 所合成目标化合
物的红外、核磁共振氢谱和碳谱、高分辨质谱谱图以及 元素分析数据. 这些材料可以免费从本刊网站(http:// sioc-journal.cn/)上下载.

\section{References}

[1] Su, S.-Q. Modern Agrochem. 2002, (3), 1 (in Chinese). (苏少泉，现代农药, 2002, (3), 1.)

[2] Beaudegnies, R.; Edmunds, A. J. F.; Fraser, T. E. M.; Hall, R. G.; Hawkes, T. R.; Mitchell G.; Schaetzer, J.; Wendeborn, S.; Wibley, J. Bioorg. Med. Chem. 2009, 17, 4134.

[3] Yang, L.; Zhang, R.-Q.; Ye, F. Modern Agrochem. 2003, (2), 1 (in Chinese). (杨丽，张荣全，叶非，现代农药, 2003，(2), 1.)

[4] Yang, J.-C.; Wu, Q.; Reng, L.-H.; Liu, C.-L. Agrochemicals 2012, 51, 547 (in Chinese) (杨吉春, 吴孤, 任兰会, 刘长令, 农药, 2012, 51, 547.)

[5] Guo, S.; Yang, F.-M.; Zhang, L. Agrochemicals 2001, 40, 20 (in Chinese).

(郭胜，杨福民，张林，农药, 2001, 40, 20.)

[6] Cheng, Z.-Z.; Wang, Y.; Gong, K.; Wang, G-H.; Li, W.-B.; Ye, L. Agrochemicals 2013, 52, 328 (in Chinese). (程正载, 王洋, 枈凯, 王光华, 李文兵, 叶龙, 农药, 2013, 52, 328.)

[7] Zou, X.-J.; Gao, S.; Lin, C.-F.; Dong, H.; Zhang, Z.-J. Agrochemicals 2005, 44, 382 (in Chinese).

(邹晓锦，高爽，林长福，董海，张宗俭，农药， 2005, 44, 382.)

[8] Liu, Y.-X.; Zhou, B.-Q.; Chai, B.-S.; Liu, C.-L. Agrochemicals 2007, 46, 649 (in Chinese).

(刘远雄, 邹本勤, 柴宝山, 刘长令, 农药, 2007, 46, 649.)

[9] Fellman, J. H. Methods Enzymol. 1987, 142, 148.

[10] Buckthal, D. J.; Roche, P. A.; Moorehead, T. J.; Forbes, B. J. R.; Hamilton, G. A. Methods Enzymol. 1987, 142, 132.

[11] Zhang, M.; Wei, J.-F.; Bai, Y.-J.; Gao, Y.; Wu, D.; Miao, Y.-Q.; Shi, Z. Chin. J. Org. Chem. 2006, 26, 207 (in Chinese).

(张敏, 魏俊发, 白银娟, 高勇, 吴亚, 苗延青, 史真, 有机化学, 2006, 26, 207.)

[12] Sui, X.-F.; Yuan, J.-Y.; Zhou, M.; He, Y.-H. Chin. J. Org. Chem. 2006, 26, 1518 (in Chinese).

(隋晓峰，袁金颖，周密，何永洪，有机化学, 2006, 26, 1518.)

[13] Nayak, M.; Batchu, H.; Batra, S. Tetrahedron Lett. 2012, 53, 4206.

[14] Markovic, V.; Eric, S.; Juranic, Z. D.; Stanojkovic, T.; Ljubinka, J. L.; Rankovic, B.; Kosanic, M.; Joksovic, M. D. Bioorg. Chem. 2011, 39, 18 .

[15] Huang, Y. Y.; Lin, H. C.; Cheng, K. M.; Su, W. N.; Sung, K. C.; Lin, T. P.; Huang, J. J.; Lin, S. K.; Wong, F. F. Tetrahedron 2009, 65, 9592.

[16] Mei, S.-K.; Chen, Y.-Z.; Gao, Z.-C. Chin. J. Appl. Chem. 1985, 2,8 (in Chinese).

(梅盛开, 程渝祖, 高忠潮, 应用化学, 1985, 2, 8.)

[17] Cui, R.-R.; Zhang, X.-Z.; Zhang, M.-F. Shangdong Pestic. News 2009 (7), 18 (in Chinese).

(崔荵荵，张秀珍，张梅风，山东农药信息, 2009, (7), 18.)

[18] Van Almsick, A.; Willms, L.; Auler, T.; Bieringer, H.; Rosinger, C. US 6376429, 2002 [Chem. Abstr. 2000, 132, 264966]. 